\title{
Natural Radioactivity at the Sin Quyen Iron-Oxide- Copper-Gold Deposit in North Vietnam
}

\author{
Dinh Chau NGUYEN ${ }^{1}$, Phon Le KHANH ${ }^{3}$, Paweł JODŁOWSKI ${ }^{2}$, \\ Jadwiga PIECZONKA ${ }^{1}$, Adam PIESTRZYŃSKI ${ }^{1}$, Hao Duong VAN ${ }^{3}$, \\ and Jakub NOWAK ${ }^{2}$
}

${ }^{1}$ AGH University of Science and Technology (AGH-UST), Faculty of Geology,

Geophysics and Environmental Protection, Krakow, Poland;

e-mail: Nguyen.Chau@fis.agh.edu.pl

${ }^{2}$ AGH University of Science and Technology (AGH-UST), Faculty of Physics and

Applied Computer Science, Krakow, Poland

${ }^{3}$ University of Mining and Geology (UMG), Hanoi, Vietnam

\begin{abstract}
The field radiometric and laboratory measurements were performed at the Sin Quyen copper deposit in North Vietnam. The field gamma-ray spectrometry indicated the concentration of uranium ranging from 5.5 to $87 \mathrm{ppm}$, thorium from 5.6 to $33.2 \mathrm{ppm}$, and potassium from 0.3 to $4.7 \%$. The measured dose rates ranged from 115 to $582 \mathrm{nGy} / \mathrm{h}$, the highest doses being at the copper ore. Concentrations in the solid samples were in the range of $20-1700 \mathrm{~Bq} / \mathrm{kg}$ for uranium, 20-92.7 Bq/ $\mathrm{kg}$ for thorium, and $7-1345 \mathrm{~Bq} / \mathrm{kg}$ for potassium. The calculated doses were from 22 to $896 \mathrm{nGy} / \mathrm{h}$; both measured and calculated dose rates are mostly related to uranium. Concentrations of radium in water samples were below $0.17 \mathrm{~Bq} / \mathrm{L}$. Uranium in water samples was significantly higher than the hydrogeological background; the maximum of $13 \mathrm{~Bq} / \mathrm{L}$ was at the waste zone pool, but neither radium nor uranium were present in tap water. Radon concentration in the dwelling air was from 42 to $278 \mathrm{~Bq} / \mathrm{m}^{3}$ for ${ }^{222} \mathrm{Rn}$ and from 8 to $193 \mathrm{~Bq} / \mathrm{m}^{3}$ for ${ }^{220} \mathrm{Rn}$. The estimated committed dose rates
\end{abstract}

Ownership: Institute of Geophysics, Polish Academy of Sciences;

(C) 2016 Nguyen et al. This is an open access article distributed under the Creative Commons Attribution-NonCommercial-NoDerivs license,

http://creativecommons.org/licenses/by-nc-nd/3.0/. 
were principally related to ${ }^{222} \mathrm{Rn}$ concentration and ranged from 1.1 to $8.1 \mathrm{mSv} / \mathrm{y}$.

Key words: IOCG deposit, natural radionuclides, dose rate.

\section{INTRODUCTION}

Iron oxide copper gold (IOCG) deposits are usually characterized by a broad spectrum of various minerals. Apart from iron and copper bearing minerals such as, e.g., magnetite, pyrite and chalcopyrite, there are also minerals containing gold, silver, rare Earth elements and natural radionuclides (Baker 2005, Corriveau et al. 2007, Hunt et al. 2005, Piestrzyński 1989, Sillitoe 2003, Williams et al. 2005, Zhao and Zhou 2011). Mining of the ore from such a deposit usually leads to an increased concentration of natural radioactive elements in the mined material which may cause a significant increase of radiation in the environment and therefore it would be a health hazard to the miners and to population of the region. To investigate this effect, both field in situ measurements and laboratory analyses of the collected samples were performed. The field survey included determination of natural radionuclides' concentration through gamma-ray spectrometry and measurements of the absorbed dose rates through gamma-ray dosimetry; concentration of radon in the air of dwellings in the vicinity of the mining area was measured using the track detectors. The field measurements and solid sample collection were performed mainly along the profile crossing the strike line of geological structure, at the active copper mining area and at the waste dumps, reservoir sediments and floatation products. Water samples were collected from the surface water pools at the open pit mine, waste dumps and from: Red River, the private wells and tap water. All the collected samples were analyzed for natural radionuclides. On the basics of the obtained data, gamma-ray dose rates and annual committed doses were estimated.

\section{REGION OF INVESTIGATION}

The Sin Quyen IOCG deposit in Lao-Cai North Vietnam is localized on the right of the Red River, which is a natural Vietnam-China boundary in this region (Fig. 1a). The deposit was discovered in 1961, owing the field radiometric and magnetic surveys. The area and copper wealth of this deposit amount to above 120 ha and 52 million tons, respectively. The principal rocks occurring at the Sin Quyen copper deposit are Paleozoic metamorphic gneiss, sandstone, mudstone, intrusive granitoid and skarn; they occur nearly vertically and trend in the NW-SE direction (Fig. 1b). The ore bodies are often met at contact zones between intrusives and sediments as lens that are several tens meters long and dozen meters thick. Since 2006 this IOCG deposit had been mined as an open pit mine. Close to the copper mine there is 


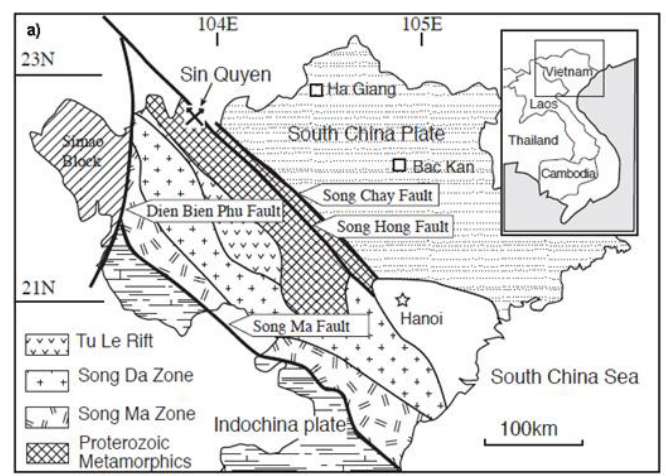

Fig. 1a. Sketch of tectonics of the Northern Vietnam and location of Sin Quyen Deposit (Ishihara et al. 2011, McLean 2001).

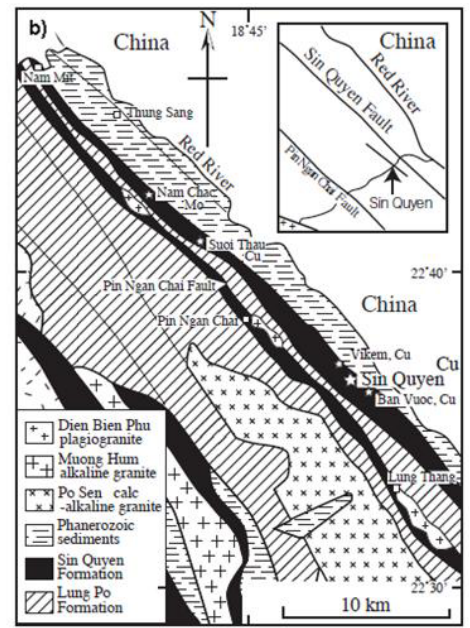

Fig. 1b. Geological map of the Sin Quyen deposit (Ishihara et al. 2011, Bui et al. 2004, McLean 2001).

floatation plant and melting factory. The annual spoil rock excavation, ore exploitation and copper metal product amount to over 6 million cubic meters, one million tons and near 12000 tons, respectively (Le et al. 2015).

\section{MEASUREMENT METHODS}

\subsection{Field measurement and sample collection}

The field radiometric measurements were performed using the 512 channels potable spectrometer GF5 with $\mathrm{NaI}(\mathrm{Tl})$ scintillation crystal of the Czech Gamma Surveyor Company ${ }^{\mathrm{TM}}$; at each measurement point the detector was placed one meter above the Earth surface. The measured data were given on display as the concentration of $\mathrm{K}[\%]$, eU [ppm], eTh [ppm], and gamma absorbed dose rate $\mathrm{D}[\mathrm{nGy} / \mathrm{h}]$. For each point the measurements were performed three times and the final results and their adequate uncertainty were estimated using the data obtained. The coordinates of the measurement points, the average concentrations and uncertainty of uranium, thorium and potassium are summarized in Table 1.

The solid samples were collected from the selected points, such as the mining waste dumps and the sediment of floatation reservoir. The samples represented the rocks and ores occurring at the studied region. Additionally, some samples were collected from the interesting places, such as high dose rate or/and high content of the natural radionuclides and so on. The mass of the solid samples varied from 0.5 to $1.0 \mathrm{~kg}$, and every sample was placed in the plastic bag. The geographic coordinates of the solid sampling places are summarized in Table 2. 
Table 1

Potassium, radium, and thorium concentrations and gamma adsorbed dose rates measured by a potable gamma spectrometer

\begin{tabular}{|c|c|c|c|c|c|c|}
\hline \multirow{2}{*}{$\begin{array}{l}\text { Point } \\
\text { No. }\end{array}$} & \multicolumn{2}{|c|}{ Coordinate } & \multirow{2}{*}{$\begin{array}{c}\mathrm{K} \\
{[\%]}\end{array}$} & \multirow{2}{*}{$\begin{array}{c}\mathrm{U} \\
{[\mathrm{ppm}]}\end{array}$} & \multirow{2}{*}{$\begin{array}{c}\text { Th } \\
{[\mathrm{ppm}]}\end{array}$} & \multirow{2}{*}{$\begin{array}{c}\text { Dose } \\
{[\mathrm{nGy} / \mathrm{h}]}\end{array}$} \\
\hline & latitude & longitude & & & & \\
\hline 1 & $22^{\circ} 36^{\prime} 45^{\prime \prime} 63$ & $103^{\circ} 48^{\prime} 54^{\prime \prime} 24$ & $2.35 \pm 0.25$ & $12.4 \pm 3.6$ & $16.8 \pm 1.8$ & $143 \pm 1$ \\
\hline 2 & $22^{\circ} 36^{\prime} 39^{\prime \prime} 00$ & $103^{\circ} 48^{\prime} 54^{\prime \prime} 27$ & $2.59 \pm 0.11$ & $8.30 \pm 0.61$ & $16.9 \pm 0.4$ & $125 \pm 6$ \\
\hline 3 & $22^{\circ} 36^{\prime} 38^{\prime \prime} 54$ & $103^{\circ} 48^{\prime} 53^{\prime \prime} 99$ & $2.69 \pm 0.09$ & $8.30 \pm 0.32$ & $14.7 \pm 1.2$ & $120 \pm 5$ \\
\hline 4 & $22^{\circ} 36^{\prime} 37^{\prime \prime} 55$ & $103^{\circ} 48^{\prime} 52^{\prime \prime} 21$ & $3.24 \pm 0.16$ & $8.00 \pm 0.75$ & $18.1 \pm 0.7$ & $134 \pm 4$ \\
\hline 5 & $22^{\circ} 36^{\prime} 37^{\prime \prime} 35$ & $103^{\circ} 48^{\prime} 51^{\prime \prime} 97$ & $2.96 \pm 0.06$ & $7.37 \pm 0.41$ & $15.8 \pm 0.4$ & $120 \pm 2$ \\
\hline 6 & $22^{\circ} 36^{\prime} 37^{\prime \prime} 45$ & $103^{\circ} 48^{\prime} 51^{\prime \prime} 76$ & $2.95 \pm 2.1$ & $11.8 \pm 1.9$ & $19.9 \pm 2.0$ & $156 \pm 7$ \\
\hline 7 & $22^{\circ} 36^{\prime} 35^{\prime \prime} 73$ & $103^{\circ} 48^{\prime} 52^{\prime \prime} 54$ & $3.56 \pm 0.6$ & $9.37 \pm 0.35$ & $23.7 \pm 3.5$ & $159 \pm 18$ \\
\hline 8 & $22^{\circ} 36^{\prime} 35^{\prime \prime} 18$ & $103^{\circ} 48^{\prime} 52^{\prime \prime} 44$ & $2.88 \pm 0.06$ & $22.9 \pm 1.1$ & $17.5 \pm 1.1$ & $212 \pm 4$ \\
\hline 9 & $22^{\circ} 36^{\prime} 35^{\prime \prime} 75$ & $103^{\circ} 48^{\prime} 50^{\prime \prime} 51$ & $2.93 \pm 0.32$ & $5.5 \pm 1.5$ & $13.0 \pm 2.7$ & $103 \pm 19$ \\
\hline 10 & $22^{\circ} 36^{\prime} 43^{\prime \prime} 29$ & $103^{\circ} 48^{\prime} 49^{\prime \prime} 50$ & $4.52 \pm 0.79$ & $10.1 \pm 2.5$ & $27.5 \pm 3.9$ & $185 \pm 33$ \\
\hline 11 & $22^{\circ} 36^{\prime} 33^{\prime \prime} 64$ & $103^{\circ} 48^{\prime} 51^{\prime \prime} 19$ & $3.66 \pm 0.07$ & $8.40 \pm 1.0$ & $22.0 \pm 2.7$ & $151 \pm 5$ \\
\hline 12 & $22^{\circ} 36^{\prime} 33^{\prime \prime} 64$ & $103^{\circ} 48^{\prime} 50^{\prime \prime} 60$ & $2.20 \pm 0.23$ & $13.5 \pm 1.5$ & $28.6 \pm 2.2$ & $177 \pm 3$ \\
\hline 13 & $22^{\circ} 36^{\prime} 33^{\prime \prime} 47$ & $103^{\circ} 48^{\prime} 49^{\prime \prime} 06$ & $2.77 \pm 0.01$ & $57.2 \pm 0.4$ & $26.8 \pm 0.3$ & $428 \pm 2$ \\
\hline 14 & $22^{\circ} 36^{\prime} 32^{\prime \prime} 91$ & $103^{\circ} 48^{\prime} 48^{\prime \prime} 54$ & $4.68 \pm 0.25$ & $7.2 \pm 0.7$ & $15.8 \pm 0.2$ & $142 \pm 7$ \\
\hline 15 & $22^{\circ} 36^{\prime} 34^{\prime \prime} 31$ & $103^{\circ} 48^{\prime} 45^{\prime \prime} 34$ & $1.55 \pm 0.15$ & $28.8 \pm 1.9$ & $21.0 \pm 1.0$ & $236 \pm 10$ \\
\hline 16 & $22^{\circ} 36^{\prime} 36^{\prime \prime} 48$ & $103^{\circ} 48^{\prime} 43^{\prime \prime} 96$ & $2.91 \pm 0.21$ & $87.1 \pm 2.5$ & $19.5 \pm 2.4$ & $581 \pm 11$ \\
\hline 17 & $22^{\circ} 36^{\prime} 35^{\prime \prime} 64$ & $103^{\circ} 48^{\prime} 44^{\prime \prime} 77$ & $2.03 \pm 0.30$ & $62.8 \pm 2.3$ & $21.5 \pm 3.1$ & $437 \pm 5$ \\
\hline 18 & $22^{\circ} 36^{\prime} 36^{\prime \prime} 15$ & $103^{\circ} 48^{\prime} 43^{\prime \prime} 19$ & $2.31 \pm 0.11$ & $55.3 \pm 1.8$ & $22.9 \pm 1.3$ & $402 \pm 8$ \\
\hline 19 & $22^{\circ} 36^{\prime} 33^{\prime \prime} 76$ & $103^{\circ} 48^{\prime} 48^{\prime \prime} 92$ & $2.82 \pm 0.06$ & $66.6 \pm 3.5$ & $22.4 \pm 1.7$ & $472 \pm 25$ \\
\hline 20 & $22^{\circ} 36^{\prime} 33^{\prime \prime} 53$ & $103^{\circ} 48^{\prime} 48^{\prime \prime} 81$ & $2.42 \pm 0.13$ & $23.7 \pm 3.4$ & $16.5 \pm 1.7$ & $208 \pm 19$ \\
\hline 21 & $22^{\circ} 36^{\prime} 33^{\prime \prime} 49$ & $103^{\circ} 48^{\prime} 48^{\prime \prime} 46$ & $3.93 \pm 0.15$ & $11.2 \pm 0.7$ & $18.4 \pm 0.8$ & $161 \pm 5$ \\
\hline 22 & $22^{\circ} 36^{\prime} 33^{\prime \prime} 46$ & $103^{\circ} 48^{\prime} 48^{\prime \prime} 43$ & $3.63 \pm 0.04$ & $10.1 \pm 1.9$ & $16.0 \pm 0.2$ & $145 \pm 10$ \\
\hline 23 & $22^{\circ} 36^{\prime} 33^{\prime \prime} 36$ & $103^{\circ} 48^{\prime} 48^{\prime \prime 2} 29$ & $4.18 \pm 0.21$ & $8.15 \pm 1.1$ & $15.0 \pm 0.5$ & $139 \pm 5$ \\
\hline 24 & $22^{\circ} 36^{\prime} 33^{\prime \prime} 20$ & $103^{\circ} 48^{\prime} 48^{\prime \prime} 40$ & $4.12 \pm 0.25$ & $7.5 \pm 0.3$ & $14.6 \pm 0.8$ & $133 \pm 1$ \\
\hline 25 & $22^{\circ} 36^{\prime} 32^{\prime \prime} 91$ & $103^{\circ} 48^{\prime} 48^{\prime \prime} 47$ & $4.7 \pm 0.29$ & $6.0 \pm 0.4$ & $6.0 \pm 0.1$ & $111 \pm 2$ \\
\hline 26 & $22^{\circ} 36^{\prime} 32^{\prime \prime} 91$ & $103^{\circ} 48^{\prime} 48^{\prime \prime 2} 29$ & $3.56 \pm 0.21$ & $12.1 \pm 1.6$ & $18.4 \pm 2.0$ & $162 \pm 11$ \\
\hline 27 & $22^{\circ} 36^{\prime} 34^{\prime \prime} 10$ & $103^{\circ} 48^{\prime} 46^{\prime \prime} 64$ & $1.90 \pm 0.19$ & $38.2 \pm 3.5$ & $33.2 \pm 5.4$ & $325 \pm 4$ \\
\hline 28 & $22^{\circ} 36^{\prime} 33^{\prime \prime} 10$ & $103^{\circ} 48^{\prime} 48^{\prime \prime} 05$ & $1.88 \pm 0.09$ & $25.4 \pm 1.3$ & $11.9 \pm 1.2$ & $200 \pm 4$ \\
\hline 29 & $22^{\circ} 36^{\prime} 32^{\prime \prime} 84$ & $103^{\circ} 48^{\prime} 48^{\prime \prime} 01$ & $3.97 \pm 0.35$ & $10.1 \pm 0.1$ & $17.9 \pm 0.6$ & $145 \pm 6$ \\
\hline 30 & $22^{\circ} 36^{\prime} 31^{\prime \prime} 96$ & $103^{\circ} 48^{\prime} 47^{\prime \prime} 18$ & $3.28 \pm 0.30$ & $12.6 \pm 2.4$ & $25.0 \pm 3.3$ & $177 \pm 12$ \\
\hline 31 & $22^{\circ} 36^{\prime} 32^{\prime \prime} 48$ & $103^{\circ} 48^{\prime} 47^{\prime \prime} 39$ & $3.36 \pm 0.29$ & $10.5 \pm 0.7$ & $14.0 \pm 0.5$ & $139 \pm 9$ \\
\hline 32 & $22^{\circ} 36^{\prime} 32^{\prime \prime} 11$ & $103^{\circ} 48^{\prime} 46^{\prime \prime} 72$ & $1.88 \pm 0.15$ & $7.8 \pm 1.1$ & $14.2 \pm 1.2$ & $105 \pm 8$ \\
\hline 33 & $22^{\circ} 36^{\prime} 31^{\prime \prime} 52$ & $103^{\circ} 48^{\prime} 46^{\prime \prime} 03$ & $2.70 \pm 0.15$ & $24.0 \pm 0.2$ & $18.5 \pm 0.1$ & $218 \pm 2$ \\
\hline 34 & $22^{\circ} 36^{\prime} 31^{\prime \prime} 69$ & $103^{\circ} 48^{\prime} 45^{\prime \prime} 96$ & $3.27 \pm 0.06$ & $9.20 \pm 1.1$ & $20.3 \pm 0.4$ & $146 \pm 10$ \\
\hline 35 & $22^{\circ} 36^{\prime} 31^{\prime \prime} 46$ & $103^{\circ} 48^{\prime} 45^{\prime \prime} 61$ & $3.26 \pm 0.29$ & $8.9 \pm 1.1$ & $24.8 \pm 6.5$ & $156 \pm 9$ \\
\hline 36 & $22^{\circ} 36^{\prime} 30^{\prime \prime} 93$ & $103^{\circ} 48^{\prime} 45^{\prime \prime} 41$ & $1.63 \pm 0.03$ & $10.4 \pm 0.8$ & $19.4 \pm 0.7$ & $129 \pm 6$ \\
\hline 37 & $22^{\circ} 36^{\prime} 30^{\prime \prime} 54$ & $103^{\circ} 48^{\prime} 45^{\prime \prime} 09$ & $3.24 \pm 0.22$ & $5.70 \pm 0.7$ & $14.6 \pm 0.6$ & $112 \pm 8$ \\
\hline 38 & $22^{\circ} 37^{\prime} 05^{\prime \prime} 25$ & $103^{\circ} 48^{\prime} 43^{\prime \prime} 85$ & $0.76 \pm 0.07$ & $24.4 \pm 1.8$ & $7.4 \pm 0.9$ & $167 \pm 11$ \\
\hline 39 & $22^{\circ} 37^{\prime} 04^{\prime \prime} 51$ & $103^{\circ} 48^{\prime} 45^{\prime \prime} 33$ & $0.38 \pm 0.05$ & $30.5 \pm 0.9$ & $5.6 \pm 0.7$ & $193 \pm 6$ \\
\hline 40 & $22^{\circ} 37^{\prime} 24^{\prime \prime} 71$ & $103^{\circ} 48^{\prime} 38^{\prime \prime} 05$ & $4.0 \pm 0.6$ & $24.0 \pm 2.4$ & $15.0 \pm 0.7$ & $227 \pm 18$ \\
\hline 41 & $22^{\circ} 37^{\prime} 27^{\prime \prime} 47$ & $103^{\circ} 48^{\prime} 28^{\prime \prime} 39$ & $2.98 \pm 0.20$ & $29.0 \pm 0.7$ & $19.9 \pm 1.1$ & $253 \pm 4$ \\
\hline \multicolumn{3}{|c|}{ Min: } & & 5.5 & 6.0 & 103 \\
\hline \multicolumn{3}{|c|}{ Max: } & 4.70 & 87.1 & 33.2 & 581 \\
\hline \multicolumn{3}{|c|}{ Mean: } & 3.04 & 19.8 & 19.0 & 199 \\
\hline
\end{tabular}


Table 2

${ }^{40} \mathrm{~K},{ }^{226} \mathrm{Ra},{ }^{232} \mathrm{Th}$ contents of the solid samples measured by gamma spectrometer coupled with HPGe detector and estimated gamma absorbed dose rates

\begin{tabular}{|c|c|c|c|c|c|c|}
\hline \multirow{2}{*}{ Sample } & \multicolumn{2}{|c|}{ Coordinate } & \multirow{2}{*}{$\begin{array}{c}{ }^{40} \mathrm{~K} \\
{[\mathrm{~Bq} / \mathrm{kg}]}\end{array}$} & \multirow{2}{*}{$\begin{array}{c}{ }^{226} \mathrm{Ra} \\
{[\mathrm{Bq} / \mathrm{kg}]}\end{array}$} & \multirow{2}{*}{$\begin{array}{c}{ }^{232} \mathrm{Th} \\
{[\mathrm{Bq} / \mathrm{kg}]}\end{array}$} & \multirow{2}{*}{$\begin{array}{c}\text { Dose } \\
{[\mathrm{nGy} / \mathrm{h}]}\end{array}$} \\
\hline & latitude & longitude & & & & \\
\hline W25 & $22^{\circ} 36^{\prime} 34^{\prime \prime} 42$ & $103^{\circ} 48^{\prime} 59^{\prime \prime} 38$ & $697 \pm 21$ & $114 \pm 3.4$ & $41.5 \pm 1.2$ & $106 \pm 3$ \\
\hline W13 & $22^{\circ} 36^{\prime} 34^{\prime \prime} 02$ & $103^{\circ} 48^{\prime} 58^{\prime} 65$ & $937 \pm 28$ & $40.5 \pm 1.2$ & $51.1 \pm 1.5$ & $88 \pm 3$ \\
\hline W15 & $22^{\circ} 36^{\prime} 32^{\prime} 05$ & $103^{\circ} 48^{\prime} 59^{\prime \prime} 19$ & $632 \pm 19$ & $128 \pm 4$ & $51.7 \pm 1.6$ & $116 \pm 4$ \\
\hline W21 & $22^{\circ} 36^{\prime} 30^{\prime \prime} 71$ & $103^{\circ} 48^{\prime} 59^{\prime \prime} 59$ & $795 \pm 24$ & $84.5 \pm 2.6$ & $58.6 \pm 1.8$ & $107 \pm 3$ \\
\hline W26 & $22^{\circ} 36^{\prime} 29^{\prime \prime} 94$ & $103^{\circ} 48^{\prime} 56^{\prime \prime} 09$ & $466 \pm 14$ & $640 \pm 19$ & $65.4 \pm 2.0$ & $354 \pm 11$ \\
\hline W20 & $22^{\circ} 36^{\prime} 32^{\prime \prime} 80$ & $103^{\circ} 48^{\prime} 51^{\prime \prime} 48$ & $637 \pm 19$ & $1699 \pm 51$ & $46.0 \pm 1.4$ & $839 \pm 25$ \\
\hline W27 & $22^{\circ} 36^{\prime} 32^{\prime \prime} 54$ & $103^{\circ} 48^{\prime} 51^{\prime \prime} 90$ & $417 \pm 13$ & $163 \pm 5$ & $33.3 \pm 1.0$ & $113 \pm 3$ \\
\hline W12 & $22^{\circ} 36^{\prime} 29^{\prime \prime} 94$ & $103^{\circ} 48^{\prime} 56^{\prime \prime} 09$ & $557 \pm 17$ & $280 \pm 9$ & $83.4 \pm 2.5$ & $116 \pm 6$ \\
\hline W23 & $22^{\circ} 36^{\prime} 32^{\prime \prime} 54$ & $103^{\circ} 48^{\prime} 51^{\prime \prime} 90$ & $382 \pm 11$ & $740 \pm 22$ & $90.6 \pm 2.7$ & $202 \pm 12$ \\
\hline W07 & $22^{\circ} 36^{\prime} 32^{\prime \prime} 61$ & $103^{\circ} 48^{\prime} 51^{\prime \prime} 62$ & $632 \pm 20$ & $401 \pm 12$ & $62.8 \pm 1.9$ & $412 \pm 8$ \\
\hline W08 & $22^{\circ} 36^{\prime} 32^{\prime \prime} 60$ & $103^{\circ} 48^{\prime} 52^{\prime \prime} 26$ & $484 \pm 15$ & $671 \pm 20$ & $75.7 \pm 2.3$ & $249 \pm 11$ \\
\hline W17 & $22^{\circ} 36^{\prime} 31^{\prime \prime} 01$ & $103^{\circ} 48^{\prime}$ & $964 \pm 29$ & $49.6 \pm 1.5$ & $66.1 \pm 2.0$ & $376 \pm 3$ \\
\hline W24 & $22^{\circ} 36^{\prime} 29^{\prime \prime} 85$ & $103^{\circ} 48^{\prime} 57^{\prime \prime} 18$ & $710 \pm 21$ & $57.7 \pm 1.7$ & $57.2 \pm 1.7$ & $102 \pm 3$ \\
\hline W16 & $22^{\circ} 36^{\prime} 30^{\prime \prime} 16$ & $103^{\circ} 48^{\prime} 5$ & $770 \pm 23$ & $44.8 \pm 1.4$ & $37.7 \pm 1.2$ & $90 \pm 2$ \\
\hline W31 & $22^{\circ} 36^{\prime} 28^{\prime \prime} 56$ & $103^{\circ} 48^{\prime} 53^{\prime \prime} 79$ & & & $92.7 \pm 2.8$ & $75 \pm 5$ \\
\hline W02 & $22^{\circ} 36^{\prime} 30^{\prime \prime} 71$ & $103^{\circ} 48^{\prime} 59^{\prime \prime} 06$ & $115 \pm 5$ & $41.7 \pm 1.3$ & $26.1 \pm 1.0$ & $145 \pm 1$ \\
\hline W28 & $22^{\circ} 36^{\prime} 29^{\prime \prime} 18$ & $103^{\circ} 48^{\prime} 5$ & $7 \pm 2$ & $30.0 \pm 1.0$ & $29.8 \pm 1.0$ & $40 \pm 1$ \\
\hline W22 & $22^{\circ} 36^{\prime} 29^{\prime \prime} 80$ & $103^{\circ} 48^{\prime}$ & $490 \pm 15$ & $68.3 \pm 2.0$ & $66.4 \pm 2.0$ & $32 \pm 3$ \\
\hline W18 & $22^{\circ} 36^{\prime} 30^{\prime \prime} 00$ & $103^{\circ} 48^{\prime} 55^{\prime \prime} 36$ & $581 \pm 18$ & $66.6 \pm 2.1$ & $49.5 \pm 1.5$ & $92 \pm 3$ \\
\hline W19 & $22^{\circ} 36^{\prime} 29^{\prime \prime} 61$ & $103^{\circ} 48^{\prime} 55^{\prime \prime} 08$ & $528 \pm 16$ & $58.0 \pm 1.7$ & $81.7 \pm 2.5$ & $84 \pm 3$ \\
\hline & $22^{\circ} 36^{\prime} 38^{\prime \prime} 01$ & $103^{\circ} 50^{\prime} 4$ & $690 \pm 21$ & $33.4 \pm 1.0$ & $26.4 \pm 1.0$ & $98 \pm 2$ \\
\hline W29 & $22^{\circ} 36^{\prime} 28^{\prime \prime} 49$ & $103^{\circ} 48$ & $390 \pm 12$ & $76.9 \pm 2.2$ & $42.0 \pm 1.3$ & $60 \pm 2$ \\
\hline W05 & $22^{\circ} 36^{\prime} 30^{\prime \prime} 18$ & $103^{\circ} 48^{\prime} 57^{\prime \prime} 28$ & $91 \pm 5$ & $302 \pm 10$ & $20.1 \pm 1.0$ & $77 \pm 5$ \\
\hline W01 & $22^{\circ} 36^{\prime} 30^{\prime \prime} 18$ & $103^{\circ} 48^{\prime} 58^{\prime \prime} 40$ & $123 \pm 5$ & $389 \pm 12$ & $25.8 \pm 1.0$ & $155 \pm 6$ \\
\hline W11 & $22^{\circ} 36^{\prime} 29^{\prime \prime} 94$ & $103^{\circ} 48^{\prime} 5$ & $136 \pm 5$ & $296 \pm 9$ & $26.2 \pm 1.0$ & $200 \pm 5$ \\
\hline W10 & $22^{\circ} 37^{\prime} 27^{\prime \prime} 54$ & $103^{\circ} 48^{\prime} 38^{\prime \prime} 65$ & $678 \pm 20$ & $583 \pm 17$ & $64.7 \pm 1.8$ & $336 \pm 10$ \\
\hline W09 & $22^{\circ} 37^{\prime} 24^{\prime \prime} 97$ & $103^{\circ} 49^{\prime} 13^{\prime \prime} 06$ & $536 \pm 16$ & $553 \pm 17$ & $66.1 \pm 1.9$ & $317 \pm 10$ \\
\hline W04 & $22^{\circ} 37^{\prime} 27^{\prime \prime} 99$ & $103^{\circ} 48^{\prime} 28^{\prime \prime} 39$ & $1345 \pm 40$ & $47.1 \pm 1.4$ & $64.1 \pm 2.0$ & $116 \pm 3$ \\
\hline W33 & $22^{\circ} 37^{\prime} 25^{\prime \prime} 23$ & $103^{\circ} 49^{\prime} 13^{\prime} 69$ & $326 \pm 11$ & $20 \pm 5$ & $31.8 \pm 1.0$ & $42 \pm 3$ \\
\hline W03 & $22^{\circ} 36^{\prime} 46^{\prime \prime} 69$ & $103^{\circ} 49^{\prime} 06^{\prime \prime} 21$ & $752 \pm 23$ & $49.3 \pm 1.5$ & $77.5 \pm 2.3$ & $100 \pm 3$ \\
\hline W06 & $22^{\circ} 37^{\prime} 02^{\prime \prime} 57$ & $103^{\circ} 48^{\prime} 50^{\prime \prime} 91$ & $663 \pm 20$ & $71.4 \pm 2.2$ & $61.2 \pm 1.9$ & $97 \pm 3$ \\
\hline W14 & $22^{\circ} 37^{\prime} 01^{\prime \prime} 74$ & $103^{\circ} 48^{\prime} 52^{\prime \prime} 39$ & $843 \pm 25$ & $202 \pm 6$ & $54.5 \pm 1.7$ & $161 \pm 5$ \\
\hline \multicolumn{3}{|c|}{ Min: } & t & 20 & 20 & 32 \\
\hline \multicolumn{3}{|c|}{ Max: } & 1345 & 1699 & 92 & 839 \\
\hline \multicolumn{3}{|c|}{ Mean: } & 560 & 254 & 54 & 161 \\
\hline
\end{tabular}


क

\begin{tabular}{|c|c|}
\hline 兑 & 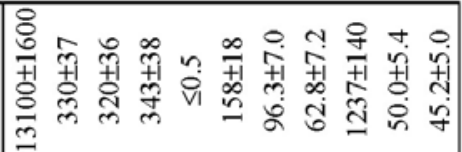 \\
\hline & 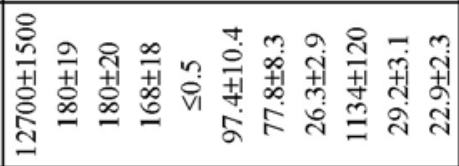 \\
\hline 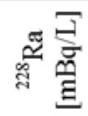 & 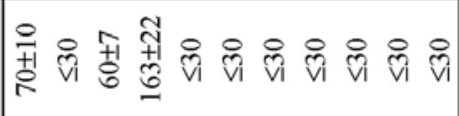 \\
\hline
\end{tabular}

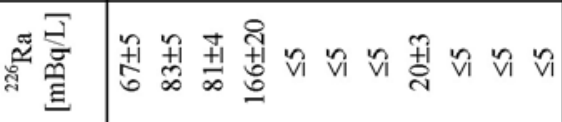

ชิ

สู

눙

สี่

๑ั

몽

î.

$\bar{\infty}$ तิ

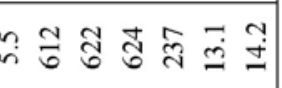

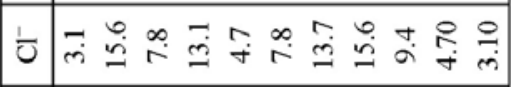

。

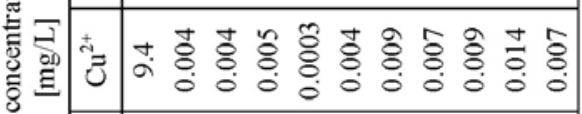

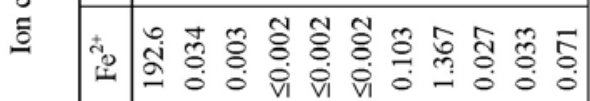

\begin{tabular}{|c|c|c|}
\hline \multirow[t]{2}{*}{ 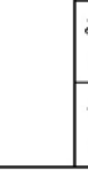 } & Uू & 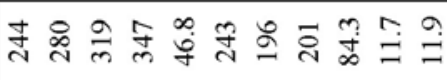 \\
\hline & + & + \\
\hline \multicolumn{2}{|c|}{ 目宣 } & 눈 \\
\hline \multicolumn{2}{|c|}{ 冤 } & 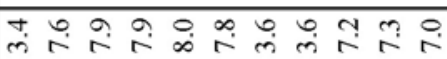 \\
\hline \multirow{2}{*}{ 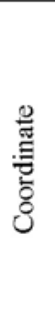 } & 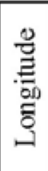 & 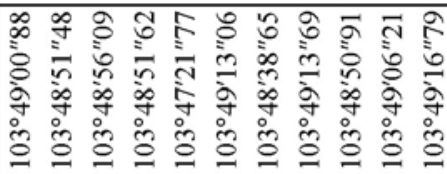 \\
\hline & 总 & 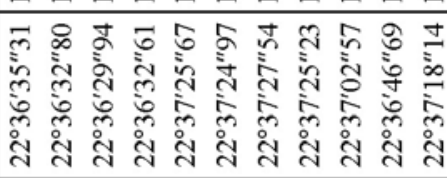 \\
\hline & & 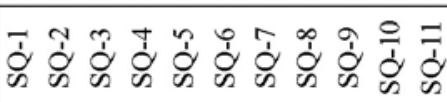 \\
\hline
\end{tabular}


The water samples were collected using a specific plastic cup and placed into $2 \mathrm{dm}^{3}$ plastic bottle. Before collecting, the cup and bottle were cleaned up using distilled water and rinsed twice by the investigated water. The coordinates of the water collecting places are presented in Table 3. All the water and solid samples were sent to AGH University of Science and Technology in Kraków, Poland.

The ${ }^{222} \mathrm{Rn}$ and ${ }^{220} \mathrm{Rn}$ concentrations in the air were measured for 22 houses surrounding the Sin Quyen IOCG deposit using Radosys detector. The Radosys detector is composed of two CR-39 track detectors and two diffusion chambers, one detector for each chamber. The detectors are designed for detecting the ${ }^{222} \mathrm{Rn}$ and ${ }^{220} \mathrm{Rn}$ activity; at every house the Radosys detec-

Table 4

Concentrations of ${ }^{222} \mathrm{Rn}$ and ${ }^{220} \mathrm{Rn}$ in the dwelling air $\left[\mathrm{Bq} / \mathrm{m}^{3}\right]$ and annual dose rate $[\mathrm{mSv} / \mathrm{a}]$ inside and outside of house

\begin{tabular}{|c|c|c|c|c|c|c|}
\hline \multirow{2}{*}{$\begin{array}{c}\text { Point } \\
\text { No. }\end{array}$} & \multicolumn{3}{|c|}{ Inner } & \multicolumn{3}{c|}{ Outer } \\
\cline { 2 - 6 }$\left[\begin{array}{c}{ }^{222} \mathrm{Rn} \\
{\left[\mathrm{Bq} / \mathrm{m}^{3}\right]}\end{array}\right.$ & $\begin{array}{c}{ }^{220} \mathrm{Rn} \\
{\left[\mathrm{Bq} / \mathrm{m}^{3}\right]}\end{array}$ & $\begin{array}{c}\text { Dose } \\
{[\mathrm{mSv} / \mathrm{a}]}\end{array}$ & $\begin{array}{c}{ }^{222} \mathrm{Rn} \\
{\left[\mathrm{Bq} / \mathrm{m}^{3}\right]}\end{array}$ & $\begin{array}{c}{ }^{220} \mathrm{Rn} \\
{\left[\mathrm{Bq} / \mathrm{m}^{3}\right]}\end{array}$ & $\begin{array}{c}\text { Dose } \\
{[\mathrm{mSv} / \mathrm{a}]}\end{array}$ \\
\hline 1 & 200 & 60 & 5.38 & 185 & 19 & 4.77 \\
2 & 165 & 40 & 4.38 & 123 & 33 & 3.28 \\
3 & 146 & 10 & 3.74 & & & \\
4 & 127 & 42 & 3.44 & 194 & 13 & 4.96 \\
5 & 278 & 36 & 7.21 & 105 & 77 & 3.08 \\
6 & 194 & 28 & 5.05 & 139 & 16 & 3.59 \\
7 & 207 & 33 & 5.40 & 110 & 9 & 2.82 \\
8 & 145 & 15 & 3.74 & 43 & 117 & 1.74 \\
9 & 193 & 23 & 4.99 & 185 & 19 & 4.77 \\
10 & 214 & 17 & 5.49 & 99 & 95 & 3.03 \\
11 & 138 & 30 & 3.65 & 177 & 88 & 4.54 \\
12 & 207 & 15 & 5.30 & 159 & 36 & 4.21 \\
13 & 191 & 22 & 4.94 & 214 & 38 & 5.61 \\
14 & 191 & 22 & 4.94 & 140 & 16 & 3.62 \\
15 & 139 & 8 & 3.55 & 163 & 13 & 4.18 \\
16 & 151 & 18 & 3.91 & 155 & 33 & 4.09 \\
17 & 163 & 13 & 4.18 & 196 & 15 & 5.02 \\
18 & 237 & 22 & 6.10 & 208 & 22 & 5.36 \\
19 & 206 & 40 & 5.42 & 175 & 74 & 4.82 \\
20 & 123 & 13 & 3.17 & 192 & 14 & 4.92 \\
21 & 42 & 193 & 2.14 & 181 & 27 & 4.71 \\
22 & 247 & 21 & 6.34 & 156 & 23 & 4.06 \\
\cline { 1 - 1 } Min: & 42 & 8 & 2.14 & 43 & 9 & 1.74 \\
Max: & 247 & 193 & 7.21 & 214 & 117 & 5.61 \\
Mean: & 177.4 & 32.8 & 4.65 & 157 & 38 & 4.15 \\
\hline
\end{tabular}


tor was hung at a height of the 1.5 to $1.8 \mathrm{~m}$ from the Earth surface and at a distance of $2 \mathrm{~m}$ from door and walls. The detectors were exposed for a period of three months (from November 2014 to February 2015). The coordinates of the investigated houses are shown in Table 4.

\subsection{Laboratory methods}

\subsubsection{Determination of uranium, thorium and potassium in a solid sample}

For gamma spectrometric measurement, the samples were milled and then dried in an oven at a temperature of $120^{\circ} \mathrm{C}$ for $24 \mathrm{~h}$ to ensure that moisture is completely removed, then accurately weighed and packed in radonimpermeable aluminums cylindrical Marinelli beakers of $720 \mathrm{ml}$ capacity and sealed to prevent escape of radon gas. The tightly sealed samples were left for at least 22 days to reach secular equilibrium between the ${ }^{222} \mathrm{Rn}$ and ${ }^{226} \mathrm{Ra}$ (Jodlowski and Kalita 2010).

The activity concentrations of ${ }^{40} \mathrm{~K},{ }^{226} \mathrm{Ra}$, and ${ }^{232} \mathrm{Th}$ were measured by gamma spectrometer coupled with a semiconductor HPGe detector (Canberra GX4020) with relative efficiency of $42 \%$ and resolution of $1.9 \mathrm{keV}$ for $1332 \mathrm{keV}$ line. The gamma spectrometer was calibrated using the IAEA reference materials RGU, RGTH, RGK as standard sources for the ${ }^{226} \mathrm{Ra},{ }^{232} \mathrm{Th}$, and ${ }^{40} \mathrm{~K}$, respectively.

The gamma lines of $1001 \mathrm{keV}$ from ${ }^{234 \mathrm{~m}} \mathrm{~Pa}$ and 609,1120 , and $1765 \mathrm{keV}$ from ${ }^{214} \mathrm{Bi}$ were used to determine the activity concentration of ${ }^{238} \mathrm{U}$ and ${ }^{226} \mathrm{Ra}$, while those of ${ }^{232} \mathrm{Th}$ were determined from the gamma lines of 911 and $969 \mathrm{keV}$ from ${ }^{228} \mathrm{Ac}$, and 583 and $2614 \mathrm{keV}$ from ${ }^{208} \mathrm{TI}$. For ${ }^{40} \mathrm{~K}$, its activity concentration was determined from its $1461 \mathrm{keV}$ gamma line. The maximum counting time for samples was 40 hours to minimize uncertainty to less than $3 \%$ for low-activity samples. The self gamma absorption resulted from the difference of density of the solid samples and standard ones was introduced follow the method described by Jodlowski (2006). The determined concentrations of ${ }^{226} \mathrm{Ra},{ }^{232} \mathrm{Th}$, and ${ }^{40} \mathrm{~K}$ are summarized in Table 2.

\subsubsection{Determination of chemical composition and radium and uranium isotopes in a water sample}

\section{Chemical composition}

The chemical composition was analyzed using an ICP-AES PerkinElmer Optima 7300 DV spectrometer, calibrated with a multi-element standard solution of the Merck ${ }^{\circledR}$ company. The induced couple plasma instrument worked with a cooling argon flow of $14 \mathrm{~L} / \mathrm{min}$, a reflected RF power of $1350 \mathrm{~W}$, both auxiliary gas and nebulizer flow rates of $1.0 \mathrm{~L} / \mathrm{min}$, a sample intake of $0.8 \mathrm{~mL} / \mathrm{min}$. The limit of determination depended on the individual element and ranged from a few to tens ppb with $3 \%$ of uncertainty. 


\section{Radium isotopes}

The radium isotopes were precipitated from the water sample of one litter together with barium element as the sulphate compound. Then the obtained sample was cleaned up and separated from the other isotopes using the procedure described by Nguyen et al. (1997). The final precipitate was placed in the glass vial $22 \mathrm{ml}$ of capacity and mixed with $12 \mathrm{ml}$ of gel scintillation cocktail produced by Perkin Elmer ${ }^{\mathrm{TM}}$ company and measured using the $\alpha / \beta$ 1414 Wallac Liquid Scintillation Counter ${ }^{\circledR}$. To eliminate the background radiation originating from the chemical reagents, cosmic and electronic noise, the background sample from the distilled water was prepared together with the series of the investigated water samples. Every sample was measured for two hours daily until the day when the expected equilibrium between ${ }^{226} \mathrm{Ra}$ and its short-lived products was established (above 21 days). The contents of ${ }^{226} \mathrm{Ra}$ and ${ }^{228} \mathrm{Ra}$ in the measured water sample were estimated on the basis of the dependence of the net measured count rates in the $\alpha$ and $\beta$ channels on the time elapsed from the precipitation radium in water sample. The limit of detection is equal to 5 and $30 \mathrm{mBq}$ per sample for ${ }^{226} \mathrm{Ra}$ and ${ }^{228} \mathrm{Ra}$, respectively. The uncertainty of the concentration of ${ }^{226} \mathrm{Ra}$ in water sample was calculated using the uncertainty of the net count rates in alpha channel of the water sample and uncertainty of the chemical efficiency obtained from the ${ }^{226} \mathrm{Ra}$ standard solution sample in accordance with the propagation law. The uncertainty of ${ }^{228} \mathrm{Ra}$ was calculated using the uncertainties of the net count rates measured in both alpha and beta channels as well as the uncertainty of the chemical efficiency. The calculation of the uncertainty for ${ }^{226} \mathrm{Ra}$ and ${ }^{228} \mathrm{Ra}$ was described in detail by Nguyen et al. (1997).

\section{Uranium isotopes}

The determination of the uranium isotopes in the water sample followed the method described by Skwarzec (1997). The uranium was precipitated from the water sample of one litter together with manganese oxide as ammonium uranyl compound. The uranium in the precipitate was separated from the other elements using $\mathrm{HCl}$ solution and ion exchange column with Dowex 100-200 mesh. Finally, the uranium in the obtained elute was again precipitated using neodymium chloride and placed onto the plastic membrane filter, $0.1 \mu \mathrm{m}$ of porosity. The obtained sample was measured using Canberra alpha spectrometer with semiconductor detector of the PIPS type. The contents of ${ }^{238} \mathrm{U}$ and ${ }^{234} \mathrm{U}$ in the water sample were estimated using the measured net count rates in the peaks of the adequate isotopes and net count rate in the peak of the ${ }^{232} U$ - an isotopic tracer. The known amount of ${ }^{232} U$ was added into water sample at the beginning of the preparation procedure. The concentration, uranium, and radium isotopes in water samples are presented in Ta- 
ble 3. The uncertainties of the concentration of ${ }^{234} \mathrm{U}$ and ${ }^{238} \mathrm{U}$ in water sample were calculated using the uncertainties of the net count rates of the peaks belonging to the ${ }^{234} \mathrm{U}$ and ${ }^{238} \mathrm{U}$ isotopes respectively, and uncertainty of the count rates of the peak belonging to the ${ }^{232} \mathrm{U}$ and uncertainty of the ${ }^{232} \mathrm{U}$ standard solution in accordance with the propagation law. The limit of detection for both isotopes $\left({ }^{234} \mathrm{U}\right.$ and $\left.{ }^{238} \mathrm{U}\right)$ amounted to $0.5 \mathrm{mBq}$ per sample.

\section{Radon isotopes}

After exposition, the track detectors were collected and transferred to the National Atomic Energy Agency of Vietnam, where the detectors were chemically treated and the ${ }^{222} \mathrm{Rn}$ and ${ }^{220} \mathrm{Rn}$ activities were determined following the Radosys procedures (Radosys 2013). The determined concentrations of radon isotopes in the air of the dwellings are summarized in Table 4.

\section{RESULTS AND DISCUSSION}

\subsection{Field gamma spectrometric and gamma absorbed dose rates}

The concentrations of uranium, thorium, potassium in the rocks and soil as well as gamma dose rates measured at the points of the profile are summarized in Table 1. The minimum, maximum, average and median values amounted to $0.4,4.7,3.0$, and $3.0 \%$ for potassium; 3.3, 87.1, 20.2, and $11.2 \mathrm{ppm}$ for uranium; 5.4, 33.2, 17.9, and $18 \mathrm{ppm}$ for thorium and 115, 581, 196 , and $159 \mathrm{nGy} / \mathrm{h}$ for gamma absorbed dose rate. The average concentrations of all mentioned elements are higher than average concentrations of the adequate element in the Earth crust, which are equal to $2.6 \%, 3.5$ and $10 \mathrm{ppm}$ for potassium, uranium and thorium respectively (Lange 1972). The average gamma absorbed dose rate at the deposit is above three times higher than the worldwide average natural dose to human $(59 \mathrm{nGy} / \mathrm{h})(\mathrm{UNSCEAR}$ 2000). The relation between the measured gamma absorbed dose rates is mostly related to the uranium concentration (Fig. 2).

\subsection{Laboratory gamma spectrometric measurements}

Table 2 summarizes the measured activity concentrations of ${ }^{40} \mathrm{~K},{ }^{226} \mathrm{Ra}$, and ${ }^{232} \mathrm{Th}$ in the solid samples. The minimum, maximum, average and median values amounted to $7,1345,560$, and $569 \mathrm{~Bq} / \mathrm{kg}$ for ${ }^{40} \mathrm{~K}, 20,1699,255$, and $99 \mathrm{~Bq} / \mathrm{kg}$ for ${ }^{226} \mathrm{Ra}$ and $20,92.7,54$, and $56 \mathrm{~Bq} / \mathrm{kg}$ for ${ }^{232} \mathrm{Th}$. The average specific activity of ${ }^{226} \mathrm{Ra}$ is six times higher than that of the Earth crust (40 Bq/kg) (UNSCEAR 2000).

The gamma absorbed dose rate was calculated based on the measured activity concentrations of ${ }^{226} \mathrm{Ra},{ }^{232} \mathrm{Th}$, and ${ }^{40} \mathrm{~K}$ in the solid samples using the following formula (UNSCEAR 2000):

$$
D[\mathrm{nGy} / \mathrm{h}]=0.041 \cdot \mathrm{K}+0.462 \cdot \mathrm{Ra}+0.604 \cdot \mathrm{Th},
$$




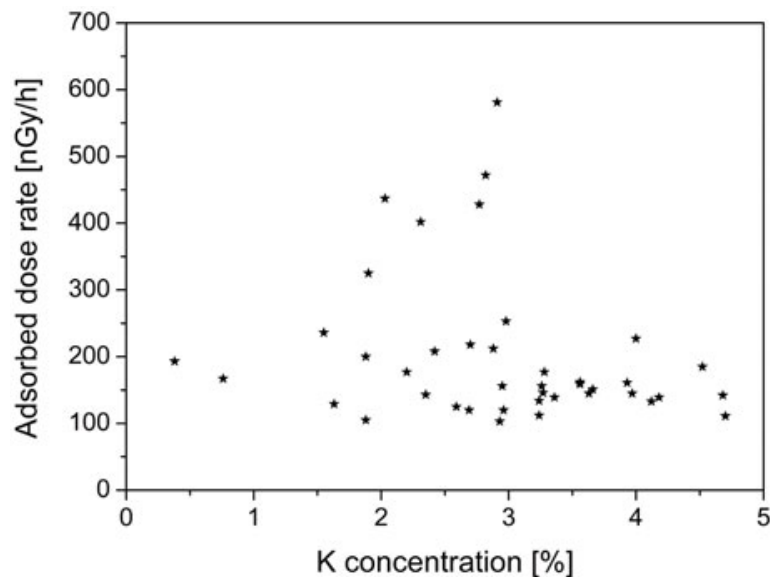

(a)

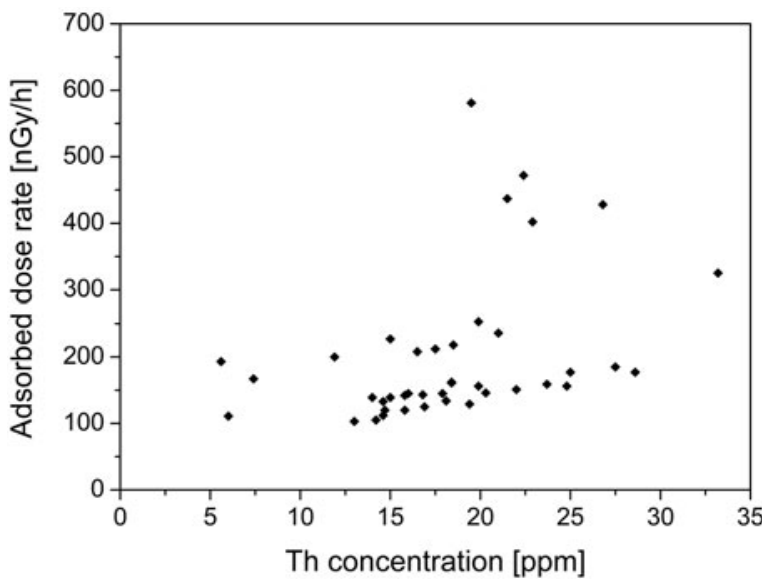

(b)

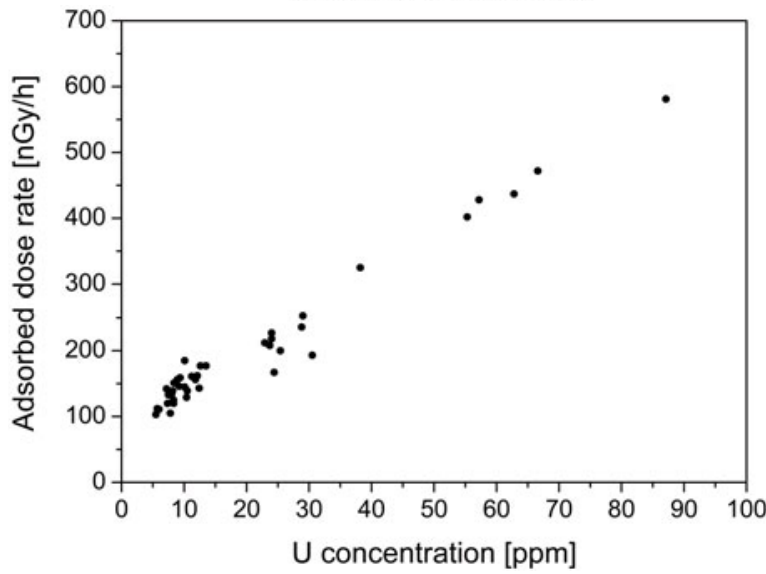

(c)

Fig. 2. Relation between the gamma absorbed dose rates and potassium (a), thorium (b) and uranium (c) measured by portable gamma spectrometer. 


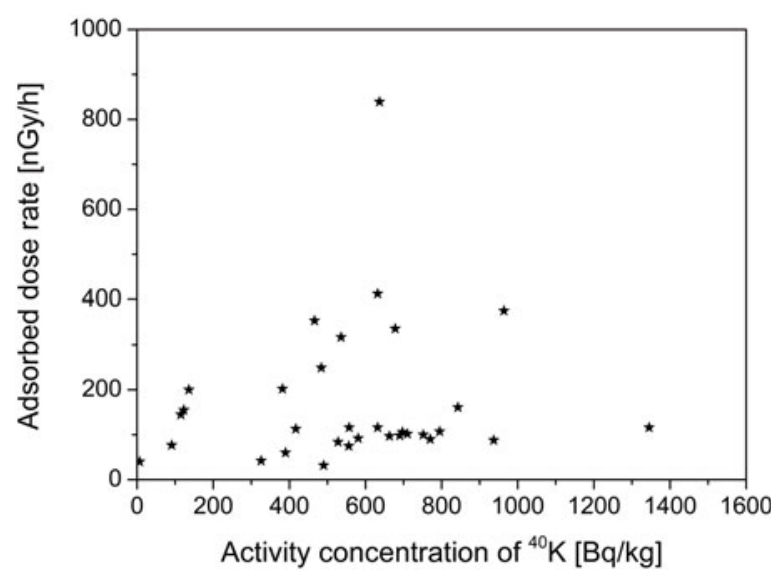

(a)

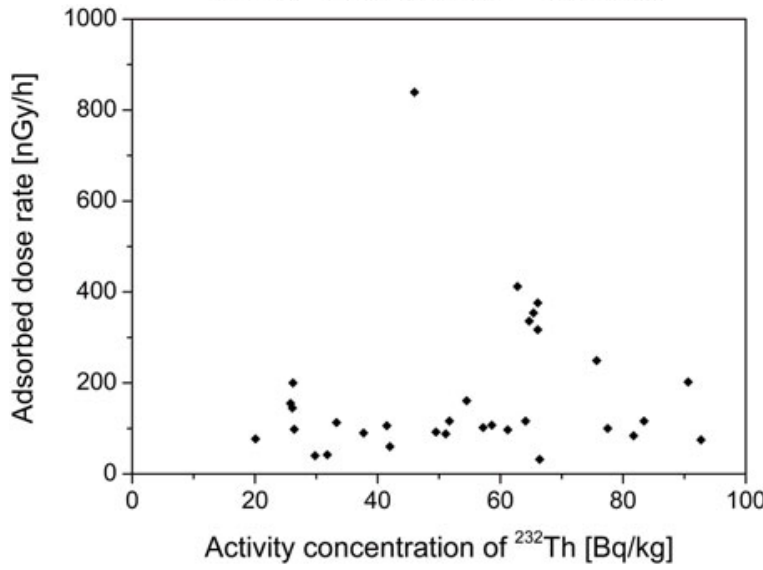

(b)

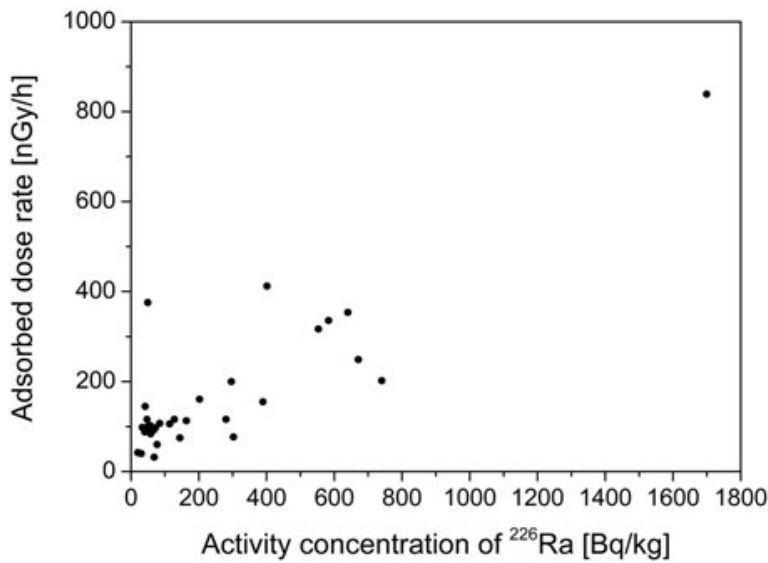

(c)

Fig. 3. Relation between estimated gamma absorbed dose rates and potassium (3), thorium (3) and uranium (3) measured by gamma spectrometer coupled with HPGe detector. 
where K, Ra, and Th are specific activities of ${ }^{40} \mathrm{~K},{ }^{226} \mathrm{Ra}$, and ${ }^{232} \mathrm{Th}$ of the sample, expressed in $\mathrm{Bq} / \mathrm{kg}$.

The calculated gamma absorbed dose rate varied from 22 to $896 \mathrm{nGy} / \mathrm{h}$ with 173 and $103 \mathrm{nGy} / \mathrm{h}$ of average and median, respectively (Table 2). The relations between estimated gamma absorbed dose rate and the activity concentrations of ${ }^{40} \mathrm{~K},{ }^{226} \mathrm{Ra}$, and ${ }^{232} \mathrm{Th}$ are presented in Fig. 3 .

Comparing Fig. 2 with adequate Fig. 3 one can see that: (i) the curves of the dependences of the gamma absorbed dose rates on the activity concentration of potassium, uranium, and thorium measured in the field are similar to those made on the basis of the data obtained from laboratory gamma-ray spectrometry measurements; (ii) the ranges of both gamma absorbed dose rates measured at the field and that calculated are comparable; (iii) the natural radioactive anomaly principally originates from the uranium occurrence in the ore and rock formations.

\subsection{Chemical composition and uranium and radium isotopes in water}

Analyzing the chemical data from Table 3, the investigated waters can be classified into several groups: (i) the waste zone water; (ii) mine water; (iii) water from the floatation reservoirs and private pool; (iv) river, and (v) tap water. The waste zone water (SQ-1) is acid $(\mathrm{pH} \sim 3.4)$ and rich in $\mathrm{Fe}^{2+}(192.6 \mathrm{mg} / \mathrm{L}), \mathrm{Cu}^{2+}(9.4 \mathrm{mg} / \mathrm{L}), \mathrm{SO}_{4}{ }^{2-}(1.85 \mathrm{~g} / \mathrm{L})$, and ${ }^{238} \mathrm{U}(12.7 \mathrm{~Bq} / \mathrm{L})$. This can be explained by long stagnation time in the contact with solid waste and atmospheric air. This condition enables to leach the iron, copper, sulphur and uranium from the pyrite, chalcopyrite and uraninite into the water. The mechanism by which pyrite is oxidized in water can be presented as follow:

$$
\mathrm{FeS}_{2}+3.5 \mathrm{O}_{2}+\mathrm{H}_{2} \mathrm{O}=\mathrm{Fe}^{2+}+2 \mathrm{SO}_{4}^{2-}+2 \mathrm{H}^{+} \text {. }
$$

The mine waters (SQ-2, SQ-3, SQ-4) are characterized by the high concentrations of radium, uranium and $\mathrm{SO}_{4}{ }^{2-}$. The water migrating along the fissures from the hill into the mine leaches the elements from the rocks. The pool and floatation reservoir waters (SQ-6, SQ-7, SQ-8) are acid $(\mathrm{pH}=3.6)$, and the $\mathrm{HCO}_{3}^{-}$concentration is very low; therefore, the waters can be regarded as the industrial water. The Red River water (SQ-9, SQ-10) is characterized by low mineralization, but it is contaminated with uranium. The significant difference in the chemical composition of tap water in comparison to the water from the Red River is the proof of its origin not from the Red River at the studied region.

\subsection{Radon isotopes}

Table 4 summarizes the concentrations of ${ }^{222} \mathrm{Rn}$ and ${ }^{220} \mathrm{Rn}$ in the air inside and outside of the dwellings. The minimum, maximum, average and median 


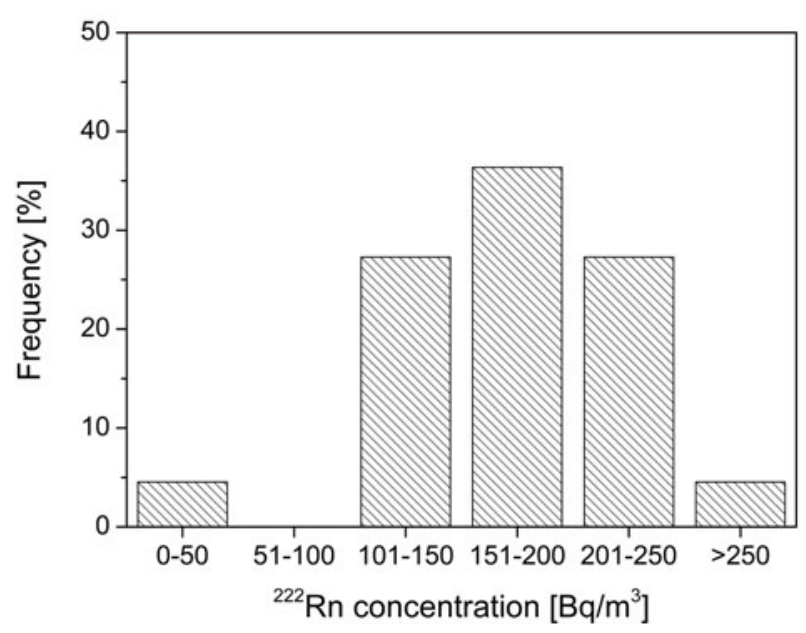

(a)

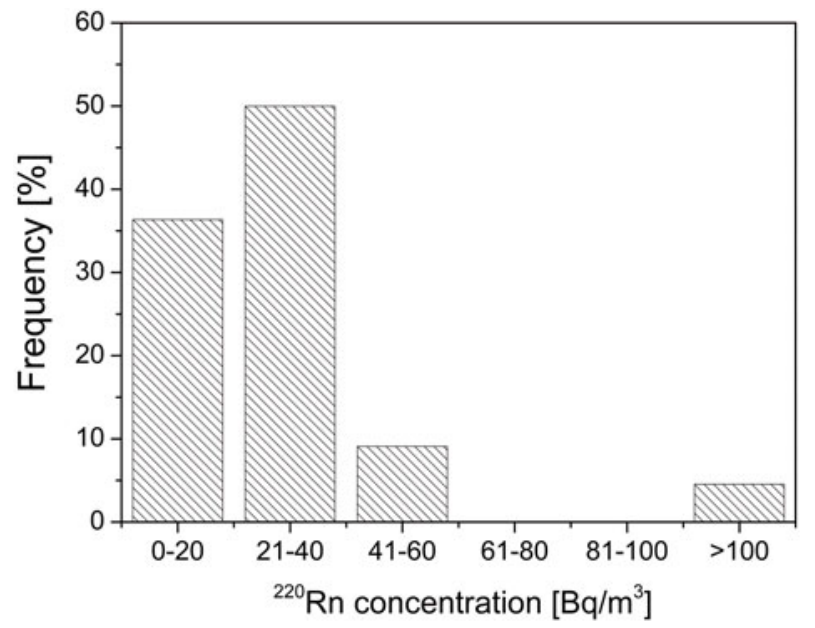

(b)

Fig. 4. Distribution of the concentrations of ${ }^{222} \mathrm{Rn}$ (a) and ${ }^{220} \mathrm{Rn}$ (b) measured in dwelling air.

values amounted to $42,278,180$, and $192 \mathrm{~Bq} / \mathrm{m}^{3}$ for ${ }^{222} \mathrm{Rn}$ and $8,193,34$, $22 \mathrm{~Bq} / \mathrm{m}^{3}$ for ${ }^{220} \mathrm{Rn}$. The concentrations of ${ }^{222} \mathrm{Rn}$ and ${ }^{220} \mathrm{Rn}$ in the air outside of the houses are similar to those inside, because the houses are often open due to tropical climate in Vietnam. The average concentration of ${ }^{222} \mathrm{Rn}$ at the Sin Quyen region is near 5 times higher than the world average $\left(37 \mathrm{~Bq} / \mathrm{m}^{3}\right)$. The contribution of the houses where the ${ }^{222} \mathrm{Rn}$ concentration was higher than $150 \mathrm{~Bq} / \mathrm{m}^{3}$ or ${ }^{220} \mathrm{Rn}$ lower than $40 \mathrm{~Bq} / \mathrm{m}^{3}$ amounted to above $70 \%$ (Fig. 4). The annual committed dose rate originated from the inhalation of ${ }^{222} \mathrm{Rn}$ and ${ }^{220} \mathrm{Rn}$ and their progeny inside and outside of the dwellings were calculated using the following Eqs. 3 and 4, respectively (UNSCEAR 2000): 


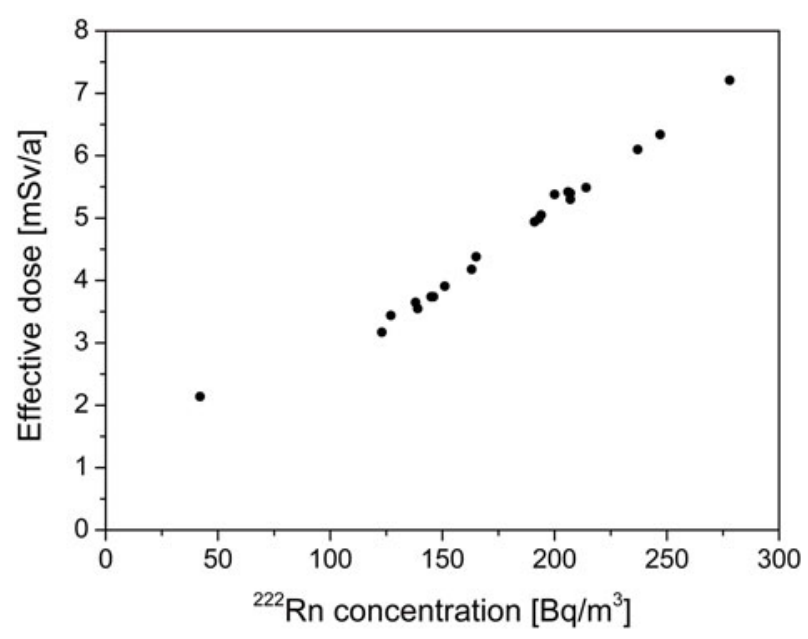

Fig. 5. Relation between estimated annual committed doses and ${ }^{222} \mathrm{Rn}$ concentration in dwelling air.

$$
\begin{aligned}
& D[\mathrm{mSv} / \mathrm{a}]=7000 \times(0.4 \times \mathrm{Rn}+0.02 \times \mathrm{Tn}) / 1000, \\
& D[\mathrm{mSv} / \mathrm{a}]=1800 \times(0.4 \times \mathrm{Rn}+0.02 \times \mathrm{Tn}) / 1000,
\end{aligned}
$$

where $\mathrm{Rn}$ and $\mathrm{Tn}$ are the concentrations expressed in $\mathrm{Bq} / \mathrm{m}^{3}$ of ${ }^{222} \mathrm{Rn}$ and ${ }^{220} \mathrm{Rn}$, respectively.

The estimated annual effective dose from radon and its progeny inhalation inside the dwellings at the Sin Quyen region ranged from 1.1 to $8.1 \mathrm{mSv} / \mathrm{a}$ with $4.45 \mathrm{mSv} / \mathrm{a}$ of average; this value is three times higher than the world average $(1.5 \mathrm{mSv} / \mathrm{a})$. The ${ }^{222} \mathrm{Rn}$ contribution in the committed dose rate amounted to above $95 \%$, and there is a linear correlation between the mentioned parameters (Fig. 5).

\section{CONCLUSION}

The Sin Quyen IOCG deposit is an elevated natural radiation area; the levels of all investigated radionuclides in the rocks, water and dwelling air are significantly higher than the adequate world averages. The average uranium concentrations measured by field survey and laboratory gamma spectrometers amount to $20.2 \mathrm{ppm}$ and $225 \mathrm{~Bq} / \mathrm{kg}$, respectively, being nearly six times higher than the average concentration in the Earth crust $(3.5 \mathrm{ppm}, 40 \mathrm{~Bq} / \mathrm{kg})$. The concentration of thorium obtained by field and laboratory measurements amounts to $17.9 \mathrm{ppm}$ and $54 \mathrm{~Bq} / \mathrm{kg}$, respectively; these values are one and haft time higher than the Earth average (10 ppm, $40 \mathrm{~Bq} / \mathrm{kg})$. The field measured and calculated gamma absorbed dose rates amount to 196 and 
$173 \mathrm{nGy} / \mathrm{h}$, while the dose rate from the natural radionuclides in the Earth crust and cosmic ration ranges from 50 to $59 \mathrm{nGy} / \mathrm{h}$ (UNSCEAR 2000).

The data of uranium in water samples show that all the surface waters are clearly contaminated with uranium isotopes; the phenomena resulted from the transformation of uranium ion from $4+$ state to $6+$ one in the contact with atmospheric air. The contents of radium isotopes in the surface waters are low; this fact can be connected with weak leaching of the radium from the rocks into the meteoric water.

The average radon concentration in the dwelling air of investigated region amounts to $169 \mathrm{~Bq} / \mathrm{m}^{3}$, being three times higher than the average world one. The annual effective dose rate from radon and its progeny inhalation amounts to $4.45 \mathrm{mSv}$ of the average. The total annual dose rate composed of the gamma radiation and radon in dwelling air is equal to $4.90 \mathrm{mSv}$.

There is a linear dependence between the gamma absorbed dose rate and uranium contents for the both field and laboratory gamma spectrometric measurements. The committed dose rate is also linearly depended on the ${ }^{222} \mathrm{Rn}$ concentration in the dwelling air. These facts confirm that the radiation anomaly at the Sin Quyen IOCG deposit is mainly generated by high uranium contents in the rocks and ores of this region.

Acknowledgment. The authors are grateful to UST-AGH Kraków for financial support, grants Nos. 11.11.140.320, 11.11.220.01, and University of Mining and Geology (UMG), Hanoi, Vietnam, grant No. 01/2012/ HD-HTQTSP.

\section{References}

Baker, T. (2005), IOCG deposits - The exploration and Research Challenges, available from: http://www.minerals.pir.sa.gov.au.

Bui, P.M., V.H. Nguyen, V.K. Phan, and D.T. Tran (2004), Geology and mineral resources of Kim Binh Lao Cai sheet. Scale 1:200 000, Dept. Geol. Mineral., Hanoi, Vietnam.

Corriveau, L., L. Ootes, H. Mumin, V. Jackson, V. Bennet, J.F. Cremer, B. Rivard, and I. McMartin (2007), Alteration vectoring to IOCG(U) deposits in frontier volcano-plutonic terrains, Canada. In: B. Milkereit (ed.), Proc. Exploration '07: Fifth Decennial Int. Conf. on Mineral Exploration, 1171-1177.

Hunt, J., T. Baker, and D. Thorkelson (2005), Regional-scale Proterozoic IOCGmineralized breccia systems: examples from the Wernecke Mountains, Yukon, Canada, Miner. Deposita 40, 492-514.

Ishihara, S., H. Hirano, M. Hoshino, N.C. Pham, T.D. Pham, and A.T. Tran (2011), Mineralogical and chemical characteristics of the allanite-rich copper and iron ore from the Sin Quyen mine, northern Vietnam, Bull. Geol. Surv. Jap. 62, 5/6, 197-209. 
Jodlowski, P. (2006), Self-absorption correction in gamma-ray spectrometry of environmental samples - an overview of methods and correction values obtained for the selected geometries, Nukleonika 51, Supl. 2, S21-S25.

Jodlowski, P., and S. Kalita (2010), Gamma-ray spectrometry laboratory for highprecision measurements of radionuclide concentrations in environmental samples, Nukleonika 55, 2, 143-148.

Lange, R. (1972), Geochemical Tables, Edition Leipzig.

Le, K.P., T.S. Nguyen, A. Piestrzynski, J. Pieczonka, P. Jodłowski, D.C. Nguyen, V.L. Nguyen, V.N. Nguyen, and H.H. Do (2015), Dispersion of radioactive materials due to processing, mining activities in Sin Quyen Copper mine, Lao-Cai, North Vietnam. In: Materials of the National Conference of the 70th Anniversary of Independence of Vietnam, 5-6 November 2015, Hanoi, Vietnam, 253-261 (in Vietnamise).

McLean, R.N. (2001), The Sin Quyen iron oxide-copper-gold-rare earth oxide mineralization of North Vietnam. In: T.M. Porter (ed.), Hydrothermal Iron Oxide Copper-Gold and Related Deposits: A Global Perspective, Vol. 2, PGC Publishing, Adelaide, 293-301.

Nguyen, D.C., J. Niewodniczański, J. Dorda, A. Ochoński, E. Chruściel, and I. Tomza (1997), Determination of radium isotopes in mine waters through alpha- and beta-activities measured by liquid scintillation spectrometry, J. Radioanal. Nucl. Chem. 222, 1, 1-2, 69-74, DOI: 10.1007/BF02034249.

Piestrzyński, A. (1989), Uranium and thorium in copper ore deposits on the foreSudetic monocline (SW Poland), Miner. Pol. 20, 1, 41-53.

Radosys (2013), Radosys User Manual of CR-39, Radosys Kft.

Sillitoe, R.H. (2003), Iron oxide-copper-gold deposits: an Andean view, Miner. Deposita 38, 7, 787-812, DOI: 10.1007/s00126-003-0379-7.

Skwarzec, B. (1997), Radiochemical methods for the determination of polonium, radiolead, uranium and plutonium in environmental samples, Chem. Anal. 42, 1, 107-113.

UNSCEAR (2000), Annex B - exposures from natural radiation sources, UNSCEAR Report 2000, 84-156.

Williams, P.J., M.D. Barton, D.A. Johnson, L. Fontbote, A. De Haller, G. Mark, N.H.S. Oliver, and R. Marschik (2005), Iron oxide copper-gold deposits: geology, space-time distribution, and possible modes of origin, Economic Geology 100th Anniv. Vol., 371-405.

Zhao, X.F., and M.F. Zhou (2011), Fe-Cu deposits in the Kangdian region, SW China: a Proterozoic IOCG (iron-oxide-copper-gold) metallogenic province, Miner. Deposita 46, 7, 731-747, DOI: 10.1007/s00126-011-0342-y.

Received 5 January 2016 Received in revised form 1 March 2016 Accepted 5 May 2016 